\title{
The new role of microRNAs in cancer
}
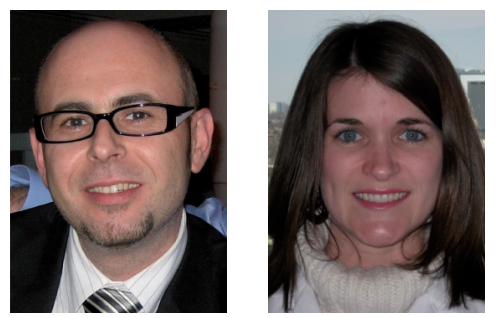

"miRNAs are emerging as major

players in complex molecular

signaling pathways, and have been

shown to be important for both

normal and diseased cell processes."

\section{Danilo Perrotti† \& Anna M Eiring}

The Ohio State University, Columbus, OH 43270, USA

${ }^{\dagger}$ Author for correspondence: 860 Biomedical Research Tower, 460 West 12th Avenue, Columbus

OH 43210, USA = Tel.: +1 6142923567 m Fax: +1 6146884181 ndanilo.perrotti@osumc.edu

The study of microRNAs (miRNAs) and their many roles in gene regulation and cancer has greatly increased over the past decade. Despite this fact, a detailed understanding of their diverse biological functions is still lacking. MiRNAs are small noncoding RNA molecules ( $19-25$ nucleotides) that bind to mRNA in a sequence-specific manner [1]. Through complementary binding to mRNA targets, primarily in the $3^{\prime}$ untranslated region (UTR), miRNAs negatively regulate protein production by either inducing mRNA cleavage, increasing mRNA decay or repressing mRNA translation [2]. miRNAs are emerging as major players in complex molecular signaling pathways [3-8], and have been shown to be important for both normal and diseased cell processes [9].

Importantly, miRNAs play critical roles in the development and progression of several types of cancers [9,10], and given the need for tightly regulated gene expression control during hematopoietic development, it is not surprising that miRNAs are important for both normal and malignant hematopoiesis [11-13]. Indeed, several miRNAs are reported to act as tumor suppressors or oncogenes in different hematologic malignancies, including acute myelogenous leukemia $[8,14,15]$, chronic myelogenous leukemia (CML) $[5,7,16,17]$, acute promyelocytic leukemia $[3,18]$, acute lymphocytic leukemia $[19,20]$ and chronic lymphocytic leukemia [21,22], among others. In all cases, gain or loss of a particular miRNA results in altered expression of an mRNA that is critical for cell maintenance, proliferation, apoptosis or differentiation. Therefore, a better understanding of the biological relevance of miRNAs in cancer will assist clinicians in the use of these tiny molecules for application in cancer diagnosis and therapy $[14,23]$.
While miRNAs are traditionally believed to negatively regulate gene expression, there are some reports in which miRNAs positively regulate gene expression $[7,24,25]$. In the first of such reports, miR-369-3 was shown to interact with two complementary sites in the 3' UTR of mRNA encoding TNF- $\alpha$, which is an AU-rich element (ARE)-containing mRNA. Upon conditions of serum starvation and cell cycle arrest, miR-369-3 recruited Argonaute 2 (Ago2) and fragile X mental retardation-related protein 1 (FXR1) to the ARE and activated its translation $[24,26]$. In the same report, they also demonstrated that the let-7 mature miRNA interacts with the 3' UTR of mRNA encoding the highmobility group protein HMGA2, and that this interaction also resulted in translational upregulation during cell cycle arrest [24]. Similar results have also been published for miRNA interactions in the 5' UTR. Indeed, miR-10a was shown to interact with the 5' UTR of ribosome protein-encoding mRNAs, resulting in positive translational regulation and consequently, enhanced ribosomal biogenesis [25].

"... a better understanding of the biological relevance of (micro) RNAs in cancer will assist clinicians in the use of these tiny molecules for application in cancer diagnosis and therapy."

In a model of CML, our group recently reported that another miRNA, miR-328, can upregulate translation of mRNA encoding the myeloid-specific transcription factor C/EBP- $\alpha[7,27,28]$. However, this case is unique from the others, because miR-328 does not directly interact with CEBPA mRNA itself, but rather with a regulatory RNA-binding protein that inhibits CEBPA translation in the acute

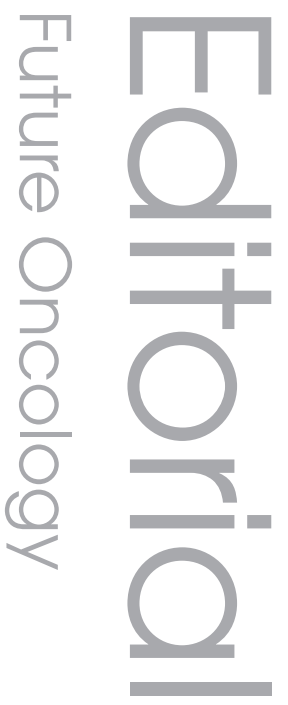


phase of this disease. CML is a clonal disorder of the pluripotent hematopoietic stem cell that is characterized by a chronic phase (CML-CP) that, if left untreated, can progress into a rapidly fatal blast crisis stage (CML-BC) [29]. In this acute stage of the disease, CML-BC patients exhibit a block of myeloid differentiation due to enhanced expression and activity of the poly $(\mathrm{rC})$-binding protein hnRNP E2 [30]. In CML-BC, but not CML-CP CD34+ bone marrow progenitors, hnRNP E2 is highly expressed and interacts with a C-rich element located in the 5' UTR of CEBPA mRNA. This interaction suppresses CEBPA mRNA translation and results in the block of differentiation observed in CML-BC ${ }^{\mathrm{CD} 34+}$ patients [30].

Interestingly, miR-328 expression is markedly reduced in $\mathrm{CML}-\mathrm{BC}^{\mathrm{CD} 34+}$ myeloid progenitors when compared with that of CML-CP patients [7], and by re-expression of miR-328 in CML-BC ${ }^{\mathrm{CD} 34+}$ progenitor cells, CEBPA mRNA translation and granulocytic differentiation are faithfully restored. However, by contrast to the other reports of miRNA-mediated translational upregulation, CEBPA mRNA does not harbor complementary binding sites for miR-328. Rather, the precursor and mature forms of miR-328 harbor C-rich regions that resemble the hnRNP E2-binding site located in CEBPA mRNA. This observation suggested that miR-328 could directly interact with hnRNP E2 and block its interaction with CEBPA mRNA, thereby restoring granulocytopoiesis of CML-BC myeloid progenitors. Using RNA electrophoretic mobility shift assays, UV-crosslinking and RNA immunoprecipitation (RIP) assays, miR-328 was shown to efficiently and specifically bind to recombinant and endogenous hnRNP E2 from CML-BC cells. Furthermore, in vitro translation assays demonstrated that synthetic miR-328 miRNA sequences impaired the inhibitory effect of recombinant hnRNP E2 on CEBPA mRNA translation, thereby increasing C/EBP- $\alpha$ protein production. Restoring miR-328 expression in CML- $\mathrm{BC}^{\mathrm{CD} 34+}$ cells through retroviral delivery completely restored C/EBP- $\alpha$ protein expression and granulocytic differentiation in response to G-CSF, and this occurred through a direct interaction between miR-328 and hnRNP E2. RNA immunoprecipitation assays further demonstrated that this interaction decreased hnRNP E2 binding to CEBPA mRNA, and quantitative real-time PCR showed that this effect was not associated with changes in total CEBPA mRNA levels. Notably, the effect of the miR-328 was independent of its seed sequence, which is the sequence responsible for canonical RNA-induced silencing complex (RISC)mediated miRNA functions. When miR-328 was mutated in its seed sequence, such that it retained its $\mathrm{C}$-rich character, the mutant form of this miRNA was still capable of binding to hnRNP E2 and restoring myeloid maturation of CML-BC progenitors. These data confirmed that the effect of miR-328 on differentiation of CML-BC cells was due to its interaction with hnRNP E2 (decoy activity) and not to downregulation of unknown miR-328 complementary mRNA targets.

“...(micro)RNAs alter mRNA metabolism not only through complementary binding to mRNA targets, but also through competition with endogenous mRNAs for binding to specific RNA-binding proteins."

In support of the notion that an RISCindependent decoy activity exists for this miRNA, the detected interaction between hnRNP E2 and miR-328 was independent of major components of the RISC complex. Indeed, co-immunoprecipitation experiments were unable to detect an interaction between hnRNP E2 with Dicer, TRBP2 or Ago2, all members of either the RISC complex or the RISC-loading complex. However, miR-328 was detected in complex with Dicer and Ago2, suggesting that, in addition to its decoy activity, this miRNA also functions in the canonical RISC-dependent manner. In fact, supplementary to its effect on myeloid maturation of CML-BC progenitors, restoration of miR-328 expression also impaired the survival and clonogenic potential of CML$\mathrm{BC}^{\mathrm{CD} 34+}$ cells. This effect was independent of its interaction with hnRNP E2, and rather occurred through canonical RISC-mediated targeting of mRNA encoding the PIM-1 serine/threonine kinase, a protein markedly upregulated in CML-BC [7,31]. PIM1 mRNA harbors a complementary miR-328 binding site in its 3' UTR that is required for miR-328 negative regulation. In fact, while the seed sequence-mutated form of miR-328 was still capable of inducing granulocytic differentiation (as previously described), it was unable to downregulate PIM-1 protein expression in CML-BC cells [7]. Altogether, these findings reveal a dual ability of miR-328 to control cell fate through both canonical basepairing with complementary mRNA targets and through direct interference with the function of 
regulatory RNA-binding proteins (decoy activity), suggesting a new mode of action for these tiny RNA molecules.

The findings of Perrotti and colleagues are disinct from all other cases of a miRNA associating with an RNA-binding protein [7], and suggest for the first time that miRNAs alter mRNA metabolism not only through complementary binding to mRNA targets, but also through competition with endogenous mRNAs for binding to specific RNA-binding proteins. It remains to be seen, however, whether additional miRNAs are also able to interact with and impair the function of regulatory RNA-binding proteins. It is conceivable that any miRNA that harbors a sequence resembling the consensus binding site of an RNA-binding protein may exhibit this sort of decoy activity. Indeed, there are several miRNAs other than miR-328 that harbor such sequences. For example, several mature human miRNA sequences harbor the AUUUA consensus binding element for AUF1 and HuR, as well as the UAGGGU consensus binding element for hnRNP A1 [Perrotti et AL., Unpublished Observations]. However, whether these miRNAs interact with and alter the RNA-binding activity of these proteins awaits further investigation. In addition to this, several other questions remain. The exact mechanism by which miR-328 is loaded onto hnRNP E2 remains unknown. Furthermore, which miRNA processing components are required for this interaction, and what subcellular compartment does the interaction initially occur? Although a reasonable assumption would be in the cytoplasm, this awaits further investigation. Future studies will be necessary to unravel the relative role of this novel interaction between miRNAs and RNAbinding proteins in other normal and malignant cell processes, as well as the potential application of this new function in cancer diagnosis and therapy.

\section{Financial \& competing interests disclosure \\ This work was supported in part by grants from the National Cancer Institute CA095512 (DP), NIH, Bethesda MD; the US Army, CML Research Program, W81XWH-07-1-0270 (DP); and by AGGRS from The OSU Graduate School (AME). Danilo Perrotti is a Scholar of The Leukemia and Lymphoma Society. The authors have no conflict of interest to disclose in relation to this work. The authors have no other relevant affili- ations or financial involvement with any organization or entity with a financial interest in or financial conflict with the subject matter or materials discussed in the manuscript apart from those disclosed. \\ No writing assistance was utilized in the production of this manuscript.}

\section{Bibliography}

1. Friedman RC, Farh KK, Burge CB, Bartel DP: Most mammalian mRNAs are conserved targets of microRNAs. Genome Res. 19(1), 92-105 (2009).

2. Bartel DP: MicroRNAs: target recognition and regulatory functions. Cell 136(2), 215-233 (2009).

3. Fazi F, Rosa A, Fatica A et al.: A minicircuitry comprised of microRNA-223 and transcription factors NFI-A and C/EBP $\alpha$ regulates human granulopoiesis. Cell 123(5), 819-831 (2005).

4. Johnnidis JB, Harris MH, Wheeler RT et al.: Regulation of progenitor cell proliferation and granulocyte function by microRNA-223. Nature 451(7182), 1125-1129 (2008).

5. Bueno MJ, Perez de Castro I, Gomez de Cedron $\mathrm{M}$ et al.: Genetic and epigenetic silencing of microRNA-203 enhances $A B L 1$ and $B C R-A B L 1$ oncogene expression. Cancer Cell 13(6), 496-506 (2008).

6. Pulikkan JA, Dengler V, Peramangalam PS et al: : Cell-cycle regulator E2F1 and microRNA-223 comprise an autoregulatory negative feedback loop in acute myeloid leukemia. Blood 115(9), 1768-1778 (2010).
7. Eiring AM, Harb JG, Neviani P et al.: miR-328 functions as an RNA decoy to modulate hnRNP E2 regulation of mRNA translation in leukemic blasts. Cell 140(5), 652-665 (2010).

8. Garzon R, Liu S, Fabbri M et al.: MicroRNA$29 \mathrm{~b}$ induces global DNA hypomethylation and tumor suppressor gene reexpression in acute myeloid leukemia by targeting directly $D N M T 3 A$ and $3 B$ and indirectly DNMT1. Blood 113(25), 6411-6418 (2009).

9. Garzon R, Calin GA, Croce CM: MicroRNAs in cancer. Annu. Rev. Med. 60, 167-179 (2009).

10. Garzon R, Fabbri M, Cimmino A, Calin GA, Croce CM: MicroRNA expression and function in cancer. Trends Mol. Med. 12(12), 580-587 (2006).

11. Chen CZ, Li L, Lodish HF, Bartel DP: MicroRNAs modulate hematopoietic lineage differentiation. Science 303(5654), 83-86 (2004).

12. Chen CZ, Lodish HF: MicroRNAs as regulators of mammalian hematopoiesis. Semin. Immunol. 17(2), 155-165 (2005).

13. Garzon R, Croce CM: MicroRNAs in normal and malignant hematopoiesis. Curr. Opin. Hematol. 15(4), 352-358 (2008).
14. Marcucci G, Maharry K, Radmacher MD et al:: Prognostic significance of, and gene and microRNA expression signatures associated with, CEBPA mutations in cytogenetically normal acute myeloid leukemia with high-risk molecular features: a Cancer and Leukemia Group B Study. J. Clin. Oncol. 26(31), 5078-5087 (2008).

15. Liu S, Wu LC, Pang J et al.: Sp1/NFkB/ HDAC/miR-29b regulatory network in KIT-driven myeloid leukemia. Cancer Cell 17(4), 33-47 (2010).

16. Venturini L, Battmer K, Castoldi $M$ et al:: Expression of the miR-17-92 polycistron in chronic myeloid leukemia (CML) CD34+ cells. Blood 109 (10), 4399-4405 (2007).

17. Agirre X, Jimenez-Velasco A, San Jose-Eneriz E et al.: Down-regulation of hsa-miR-10a in chronic myeloid leukemia CD34 $4^{+}$cells increases USF2-mediated cell growth. Mol. Cancer Res. 6(12), 1830-1840 (2008).

18. Garzon R, Pichiorri F, Palumbo T et al.: MicroRNA gene expression during retinoic acid-induced differentiation of human acute promyelocytic leukemia. Oncogene 26(28), 4148-4157 (2007). 
19. Kotani $\mathrm{A}, \mathrm{Ha} \mathrm{D}, \mathrm{Hsieh} \mathrm{J}$ et al.: miR-128b is a potent glucocorticoid sensitizer in MLL-AF4 acute lymphocytic leukemia cells and exerts cooperative effects with miR-221. Blood 114(19), 4169-4178 (2009).

20. Kotani A, Ha D, Schotte D, den Boer ML, Armstrong SA, Lodish HF: A novel mutation in the miR-128b gene reduces miRNA processing and leads to glucocorticoid resistance of MLL-AF4 acute lymphocytic leukemia cells. Cell Cycle 9(6), 6 (2010).

21. Calin GA, Dumitru CD, Shimizu M et al.: Frequent deletions and down-regulation of micro- RNA genes miR15 and miR16 at 13q14 in chronic lymphocytic leukemia. Proc. Natl Acad. Sci. USA 99(24), 15524-15529 (2002).

22. Calin GA, Liu CG, Sevignani C et al:: MicroRNA profiling reveals distinct signatures in B cell chronic lymphocytic leukemias. Proc. Natl Acad. Sci. USA 101(32), 11755-11760 (2004).
23. Kota J, Chivukula RR, O'Donnell KA et al.: Therapeutic microRNA delivery suppresses tumorigenesis in a murine liver cancer model. Cell 137(6), 1005-1017 (2009).

24. Vasudevan S, Tong Y, Steitz JA: Switching from repression to activation: microRNAs can up-regulate translation. Science 318(5858), 1931-1934 (2007).

25. Orom UA, Nielsen FC, Lund AH: MicroRNA-10a binds the 5'UTR of ribosomal protein mRNAs and enhances their translation. Mol. Cell 30 (4), 460-471 (2008).

26. Vasudevan S, Steitz JA: AU-rich-elementmediated upregulation of translation by FXR1 and Argonaute 2. Cell 128(6), 1105-1118 (2007).

27. Beitzinger M, Meister G: Preview. MicroRNAs: from decay to decoy. Cell 140(5), 612-614 (2010).
28. Gough NR: Double-duty microRNAs: destroy and decoy. Science Signaling 3(112), EC71 (2010).

29. Calabretta B, Perrotti D: The biology of CML blast crisis. Blood 103(11), 4010-4022 (2004).

30. Perrotti D, Cesi V, Trotta R et al:: BCR-ABL suppresses $\mathrm{C} / \mathrm{EBP} \alpha$ expression through inhibitory action of hnRNP E2. Nat. Genet. 30 (1), 48-58 (2002).

31. Nieborowska-Skorska M, Hoser G, Kossev P, Wasik MA, Skorski T: Complementary functions of the antiapoptotic protein $A 1$ and serine/ threonine kinase pim-1 in the $B C R /$ $A B L$-mediated leukemogenesis. Blood 99(12), 4531-4539 (2002). 